\title{
Warehouse Location Problem in Context of Delivery Time Shortening
}

\author{
Nina Kudláčkováa ${ }^{1, *}$, and Jan Chocholáć ${ }^{1}$ \\ ${ }^{1}$ University of Pardubice, Faculty of Transport Engineering, Studentská 95, 53210 Pardubice, Czech \\ Republic
}

\begin{abstract}
The current trend in logistics and the distribution chain is the pressure to reduce the delivery time, which was triggered by the e-commerce trend. To shorten the delivery time, it is necessary to choose the location of the warehouse or distribution center to maximize efficiency. Therefor is this article focused on warehouse location problem and chosen methods to solve this problem. It is also focused on use of the multi-criteria method to evaluate the alternatives for the warehouse location because it will be necessary to go even further in this area and find a combination of existing models or find new one which will be based not on costs but on delivery time. That will help companies solve the allocation problem to meet the requirements for shorter delivery times.
\end{abstract}

\section{Introduction}

Most companies are currently either directly or at least indirectly involved in a trading system that we call global. The global concept of business is quite challenging, and businesses are challenging how to manage the corresponding processes with products from the perspective of production, distribution and logistics.

The current trend in logistics and the distribution chain is the pressure to reduce the delivery time, which was triggered by the e-commerce trend.

This is a change in the delivery requirements of customers, where standard delivery is performed within 1-2 days or in the shortest possible time, free or almost free of charge.

Such a market trend needs to be adapted to the behavior of the whole logistics chain, because supply speed can be an important factor in gaining a competitive advantage.

To shorten the delivery time, it is necessary to choose the location of the warehouse or distribution center to maximize efficiency. This is an important strategic decision that has a long-term impact on the further development of the supply-demand chain.

Very important attribute that affects the supply-chain efficiency aspects is the number, size and location of the distribution centers, as well as the decision from which distribution center to serve a given range of consumers or customers.

Based on the importance of this logistical problem, a great deal of research has been devoted to the development of mathematical models to find a suitable location for the distribution center. However, most of them are based on cost minimization.

* Corresponding author: Nina.Kudlackova@upce.cz 
Current trends, however, place particular emphasis on shortening the delivery time, even at the expense of higher costs. It is therefore necessary to shift current models of decision making on the location of the warehouse network from minimizing costs to minimizing delivery times.

This raises the need to find in the current spectrum of theories of geography experts such models that are based both on distance assessment and on minimizing costs.

The decision making process for location of the warehouse network could be based on the models below.

\section{Globalization and global logistics}

While Rodrigue defines globalization simply as a term commonly used to express the mutually evolving economic, social and cultural relationships between individual world regions, The International Monetary Fund defines globalization in several levels. In the most general it characterizes it as a force that is increasingly affecting the lives of people of all countries in the world. More specifically, it defines globalization as the historical process, the result of human innovation and the technological process. In the opinion of the International Monetary Fund, it refers to the growing integration of economies around the world, particularly through the movement of goods, services and capital across borders. The IMF also recognizes the globalization of another plane, namely cultural, political and environmental $[1,2]$.

Globalization has caused many problems in logistics. For example, there have been prolonged delivery times, especially since transition times have become uncertain. There has been a significant increase in break-bulk items and also an increase in consignment consolidation requirements. In some companies, there has been a direct conflict between the process of globalization and the introduction of just-in-time (JIT) procedures under the new global strategy.

Global companies tend to prolong delivery times and inventory levels due to the large distances of the markets and the complexity of logistics operations. In companies doing business on the JIT philosophy is the situation opposite aiming at shortening delivery times and stock levels as much as possible.

The solution is the level of compromise between the cost of order fulfillment and stockholding and the costs resulting from the uncertainty of product deliveries. Thus, there is a general shift in competition. They no longer compete with companies as such, but with their distribution chains. The solution is therefore in solving the warehouse location problem and optimizing the distribution strategy $[1,3]$.

\section{Chosen methods of warehouse location problem}

The creation of a warehouse or distribution center model can be understood as a classical locational task in the field of operational theory of graph theory. As Cura states, it is possible to divide the localization tasks into static and dynamic. Static localization tasks focus more on space, dynamic to time factor $[4,11]$.

\subsection{Weber model}

The Weber model is focused on minimizing the transport costs of the company. This model defined so-called localization factors, such as raw materials or labor costs.

The so-called Weber's problem is a typical example of solution with one service center. The purposeful function for optimal solution of these tasks is expressed by equation (1): 


$$
\min Z=\Sigma_{(i \epsilon F)}=w_{i} \cdot d\left(X, P_{i}\right)
$$

The function minimizes the total cost $Z$.

A set of all demanding customers is marked as $F$, scale weights transferred on the customer costs $i$ are marked as $w_{i} . X$ a $P_{i}$, and represent searched position of the service center and the customer's position $i$, so $d\left(X, P_{i}\right)$ expresses the distance between the two locations.

The task with one operator center can be converted to task with more centers. The new form describes the following equation (2).

The set of all demanding customers is marked $\mathrm{D}$, the rated weight of the connection between the customer $i$ and the service center $j$ is, then $w_{i j}, d\left(X_{j}, P_{i}\right)$ is the distance of the searched service center $j$ and given customer $i$.

$$
\min Z=\Sigma_{(i \epsilon F)} \Sigma_{(j \in D)} w_{i j} \cdot d\left(X_{j}, P_{i}\right)
$$

By extending the task of evaluating the weight of the connection between the individual service centers we get the equation of the purpose function (3), where $v_{i k}$ indicates the weight between the service center $i$ and $k$.

$$
\min Z=\sum_{(i, k \in D, i<k)} v_{i k} \cdot d\left(X_{i}, X_{k}\right) \sum_{(i \epsilon F)} \Sigma_{(j \epsilon D)} w_{i j} \cdot d\left(X_{j}, P_{i}\right)
$$

In addition to the previous tasks, the locational allocation task, in addition to the previous tasks, optimally assigns customers to the optimally deployed service centers, provided that their demand is met.

The analytical model of the optimal solution can be formulated using the following purpose function (4) and the set of limiting conditions (5) - (9).

$$
\begin{array}{r}
\min Z=\sum_{(i \epsilon F)} \sum_{(j \epsilon D)} c(i, j) \cdot y_{i j} \\
\sum_{(j \epsilon F)} x_{j}=p \\
\sum_{(j \epsilon F)} y_{i j}=1 \forall i \epsilon D \\
x_{j \geq} y_{i j} \forall i \epsilon D, \forall j \in F
\end{array}
$$

$x_{j}=\{1$, if we place a center at one of the possible locations $j, \forall j \in F ; 0$, other situations $\}$

$y_{i j}=\{1$, if one of the available places $j$ serve the customer $i, \forall i \in D, \forall j \in F ; 0$, other situations $\}$

$F$ represents the set of possible locations of the serving point (which is called depo), $D$ represents the set set of demanding customers; $c(i, j)$ costs associated with assigning the customer $i$ to the service location $j$. The condition (5) expresses that the $p$ service points are open, the condition (6) ensures the assignment of each customer, and the limiting condition (7) allows customers to be assigned only to those service locations which are in operation. The restrictive conditions (8) and (9) define binary conditions for variables [5-7]

\subsection{Uncapacitated warehouse location problem}

As Michel \& Van Hentenryck states, the uncapacitated warehouse location problem is simply defined as a network of $\mathrm{W}+\mathrm{S}$ knots and W.S link nodes, where $\mathrm{S}$ is number of trades and $\mathrm{W}$ is number of warehouse candidates. [8]

Each warehouse has certain fixed costs $f_{i} \geq 0$, and the costs of transport from the warehouse to the store are marked as $c_{j i} \geq 0$. 
The task is to find a subset of warehouses and store assignments to stores so that total fixed costs and transport costs are minimized. This task can be simplified to simply location of subset of warehouses, while each trade should of course be assigned to a particular warehouse and that to the one that has minimum shipping costs for the store. In other words, this model determines a set of warehouses as opened, and each store will be assigned by single store in that set where $c_{j i}$ will be minimal.

This means that the decisive variable $x_{i} \in\{0,1\} i=1, \ldots \ldots, W$ (12) means, that the store $\mathrm{i}$ is opened, when $x_{i}=1(11)$, and otherwise it is closed. The mathematical model for solving this problem is, according to experts Michel and Van Hentenryck, formulated as the following (10):

$$
\begin{gathered}
\min \sum_{(i=F ; W)} f_{i} \cdot x_{i}+\sum_{(j=1 ; S)} \min \left[\left(1-x_{i}\right) \cdot M+C_{j i}\right]\left(X, P_{i}\right) \\
\sum_{(i=1 ; W)} x_{i}=1 \\
\sum_{(i=1 ; W)} x_{i} \in\{0,1\} i=1, \ldots \ldots, W
\end{gathered}
$$

$\mathrm{M}$ stands for a large enough number, such as $\mathrm{C}_{\max }+1$ is, for example $[8,9]$.

\subsection{P-median model}

The selection of the location of the warehouse is basically a selection and subsequent assignment of distribution center in a certain economic area, which is characterized by a certain degree of certain demand.

Network distribution analysis is one of the first steps in choosing a location for the center. The role of assignment of placement by the P-median model is, according to Huifenga and Aigonga, explained as follows: the number of supply stations $P$ is chosen to satisfy $\mathrm{n}$ requests from the total number of $\mathrm{m}$ supply stations in order to minimize distance, time or cost.

The assumption is the demand $i$ where $\omega_{i}$ is the quantity (weighted value) of this demand $\mathrm{i}$ and $\mathrm{d} \_\mathrm{ij}$ is the distance between the supply station $j$ to the demand $i$. The formula is as follows (13):

$$
\min \left(\Sigma_{(i=1 ; n)} \Sigma_{(j=1 ; m)} \omega_{i} \cdot \alpha_{i j} \cdot d_{i j}\right)
$$

And at the same time

$$
\begin{gathered}
\Sigma_{(j=1 ; m)} \alpha_{i j}=1, i=1,2, \ldots, n \\
\sum_{(j=1 ; m)}\left(\Pi_{(i=1 ; m)} \alpha_{i j}\right)=P, P \leq m \leq n
\end{gathered}
$$

$\alpha_{i j}$ is distribution coefficient (14) and (15), which says that if the demand $i$ is satisfied by the supply station $j$, the value of this coefficient is 1 , otherwise it is 0 .

The formula is as follows (16):

$$
\alpha_{i j}=\{1, i \text { is suppliled through } j ; 0 \text {, other situations }\}
$$

This basic storage location model always includes only individual factors however there are many factors to select the current location of the warehouse, so the use of this model would be inadequate $[9,10]$. 


\section{Storage location model minimizing delivery costs and warehouse maintenance}

In the Warehouse location problem, according to Thomas Nagy, the company is generally considering opening a warehouse in certain potential (candidate) locations to supply their existing stores.

Each possible warehouse has the same maintenance costs and the capacity is the maximum number of stores the store can supply.

Each store must be supplied with just one open stock.

In practice, the fact is that the cost of delivering goods to a store depends on the location of the warehouse.

The aim of this model is to determine which warehouses to open and which of these stores should supply different stores so that the sum of warehouse maintenance and delivery costs is minimized.

Let $\mathrm{i}$ be the number of stores, $\mathrm{j}$ express the number of stores, $\mathrm{Cij}$ is the table containing the costs associated with the supply, Sij matrix of indicators in case the store $\mathrm{i}$ is supplied in stock $\mathrm{j}$, cf represent fixed costs, and $\mathrm{Oj}$ is a vector of truth value indicating which warehouses are open.

The model is as follows (17):

$$
\min \Sigma_{(i, j)} S_{i j} \cdot c_{i j}+d_{f} \cdot \Sigma 0_{\mathrm{j}}
$$

Condition that the trade must always have one store (18):

$$
\forall_{i} \Sigma_{(j)} S_{i j}=1
$$

Condition that the warehouse has a certain capacity (19):

$$
\forall_{i} \Sigma_{(j)} S_{i j} \leq \text { Capacity } j
$$

A condition that the trade may only be stocked on stock, when the store is opened (20):

$$
\forall_{i, j} \Sigma S_{i j} \leq 0_{j}
$$

As can be seen from the above selected models, the traditional approach to addressing the warehouse location problem is to minimize costs [7, 11].

For example, according to Michel and Van Hentenryck, these models are able to simultaneously find a selection of suitable devices and meet the requirements of a given number of customers to minimize total cost of placement, operation and transport [8].

However, most cost-minimization approaches are addressed at the level of supply companies and do not include customer benefits. More advanced models addressing the warehouse location problem are based on maximizing profits. Again, no attention is paid to the customer's requirements, but this does not necessarily have to be met $[8,9]$.

In several other models, customization features such as product availability, delivery frequency, and certain elements of delivery time requirements can be found but it is still not enough. It seems we will have to link existing models of find a new one, which will reflect customization and will lead to delivery time shorting, because above mentioned trend is further developing

The decision to place the warehouse is still one of the most important decisions when creating a new or expanding existing distribution network, however, companies increasingly take the customer's wishes into account and transfer the issue to distribution and logistics centers rather than to their own supply and demand chain. 


\section{Conclusion}

As can be seen from the above, none of these models are currently tailored to meet the conditions of globalization and customer requirements.

No comprehensive systematic research framework can be found in the current literature to address the location of the distribution equipment so that it meets the customer's requirements to shorten the delivery time while meeting the requirements of the company. Many models created for the warehouse placement do not represent the consequences of criterion dependency. Most multi-criteria decision-making methods are based on the concept of adding a criterion to the assumption of independence, but in reality, not always all criteria are independent.

Multi-criteria decision models based on the use of the Choquet integrator with the evaluation of criteria by the degree of veracity and the subsequent evaluation of alternative locations for the location of the warehouse in order to determine the main and partial criteria and set the hierarchy of the warehouse location problem and then use the multicriteria method to evaluate the alternatives for the warehouse location are a step in the right direction, however, it will be necessary to go even further and find a combination of existing models and find those one which will be based not on costs but on delivery time. That will help companies solve the allocation problem to meet the requirements for shorter delivery times.

This paper is supported by the research project "From horse-drawn railway to intermodal transport" within Visegrad Fund.

\section{References}

1. J.P. Rodrique, The geography of transport system (New York, Hofstra University, USA, 2013)

2. International Monetary Fund, Globalization and Inequality (2007), Available online: http://www.imf.org/external/pubs/ft/weo/2007/02/pdf/c4.pdf

3. R. Farahani, R. Zanjirani, S. Rezapour, L. Kardar, Logistics Operations and Management - Concept and Models, 469 p. (Waltham, USA: Elsevier, 2011)

4. T. Cura, Computers \& Industrial Engineering 37, 5, 3943-3952 (2010)

5. A. Máca, L. Kalecký, Location - alocation role and centrality, 271-276 (Think Together 2013, Prague: Česká zemědělská univerzita, Czech Republic, 2013)

6. T. Demirel, N.C. Demirel, C. Kahraman, Expert Systems with Applications, 39433952 (2010)

7. S. Nickel, Location Theory - Discrete Location Problems, Facility Location and Strategic Supply Chain Management, (Saarland University, Germany, 2013)

8. L. Michel, P. Van Hentenryck, European Journal of Operational Research 157, 3, 576591 (2004)

9. T. Özcan, N. Celebi, E. Sakir, Expert Systems with Applications 38, 8, 9773-9779 (2011)

10. J. Huifeng, X. Aigong, The International Archives of the Photogrammetry, Remote Sensing and Spatial Information Sciences, XXXVII.(B2), 545-548 (2008)

11. T. Nagy, Warehouse location problem - CSP model, Available online: http://www.bitlib.net/show.php?id=3369316 (2004) 